Design Individual trajectories of height, weight and adiposity were modelled from birth to 10 years using random-effects linear-spline models. Adiposity was modelled as ponderal index $\left(\mathrm{PI}, \mathrm{kg} / \mathrm{m}^{3}\right)$ from birth to 2 years and body mass index $\left(\mathrm{kg} / \mathrm{m}^{2}\right)$ from 2 to 10 years. Interactions between self-reported maternal and paternal smoking during pregnancy (any vs none) with trajectories of height, weight, and adiposity were examined with and without adjustment for potential confounders and mutual adjustment for maternal/paternal smoking.

Setting South-West England.

Participants $\mathrm{N}=12684$ children for height models, 12773 for weight models, 12531 for PI models, and 11588 for body mass index models.

Results Maternal smoking during pregnancy had a strong impact on birth length and weight (eg, reduction in birth length in boys $0.70 \mathrm{~cm}, \mathrm{SE}$ 0.07). These effects reduced only slightly with adjustment for confounders and paternal smoking during pregnancy. Paternal smoking was associated with much smaller reductions in birth length and weight (eg, reduction of birth length in boys $0.21 \mathrm{~cm}$, SE 0.07), and associations were completely attenuated by adjustment for confounders and maternal smoking in pregnancy. There was some evidence that parental smoking during pregnancy affected height growth in later infancy (3-10 months) and weight gain in early and later infancy (0-4 and 4-11 months), with offspring of parents who smoked growing and gaining weight faster than offspring of non-smokers. Associations were stronger for maternal than paternal smoking. Height growth and weight gain in later childhood was not associated with smoking in pregnancy. The offspring of parents who smoked had lower PI at birth (eg, reduction in PI at birth for maternal smoking in pregnancy among boys $-0.25 \mathrm{~kg} / \mathrm{m} 3, \mathrm{SE} 0.08$ ), with maternal smoking having a stronger association than paternal smoking. However, associations of both maternal and paternal smoking during pregnancy on PI at birth were completely removed by adjustment for confounders. Neither maternal nor paternal smoking was associated with adiposity trajectories.

Conclusion There is evidence of an intrauterine effect of maternal smoking on birthweight and length and on height and weight growth in infancy (but not later childhood). Maternal smoking in pregnancy does not seem to affect adiposity at birth or change in this during infancy or childhood through intrauterine mechanisms.

\section{AN ANALYSIS OF THE EFFECT OF INTRAUTERINE GROWTH RETARDATION ON SPIROMETRIC LUNG FUNCTION MEASURES OF 8-YEAR-OLD CHILDREN}

doi:10.1136/jech.2010.120956.60

${ }^{1} \mathrm{~S}$ Kotecha, ${ }^{2} \mathrm{~W} \mathrm{~J}$ Watkins, ${ }^{3} \mathrm{~J}$ Heron, ${ }^{4} \mathrm{~J}$ Henderson, ${ }^{2} \mathrm{~F} \mathrm{D}$ Dunstan, ${ }^{1 S}$ Kotecha. ${ }^{1}$ Department of Child Health, School of Medicine, Cardiff University, Cardiff, UK; ${ }^{2}$ Department of Primary Care and Public Health, School of Medicine, Cardiff University, Cardiff, UK; ${ }^{3}$ Avon Longitudinal Study of Parents and Children, Department of Social Medicine, University of Bristol, Bristol, UK; ${ }^{4}$ Department of Community-Based Medicine, University of Bristol, Bristol, UK

Objective Increasing evidence suggests that intrauterine growth retardation (IUGR) is associated with long-term morbidities, including respiratory outcomes. The relationship between IUGR and lung function has been studied in adults, but few studies have investigated childhood respiratory outcomes and it is unclear if catch-up growth in these children influences lung function. We investigated the hypothesis that lung function differs in 8- to 9-yearold children born at term between those that were within normal weight bands and those that had growth retardation. Additionally, in the growth-retarded group, we investigated if lung function differed between those who did and those who did not show weight catch up.
Design The Avon Longitudinal Study of Parents and Children birth cohort.

Participants The 5770 Caucasian singleton births of 37 weeks or longer gestation who had lung function assessed at 8-9 years. We classified 576 infants as IUGR as their gestation-appropriate birthweight fell below the 10th centile. This group was compared with those 3462 non-IUGR infants whose birthweights fell between the 20 th and 80th centiles.

Main outcome measure and results The non-IUGR infants had significantly better lung function at 8-9 years of age than those with IUGR. The differences between the standardised ( $z$ ) lung function values, adjusted for sex, gestation, maternal smoking during pregnancy, and social class, and 95\% CI were FEV1: -0.198 ( -0.294 to -0.102$)$; FVC: -0.131 ( -0.227 to -0.036$)$; FEF $25-75$ : -0.149 ( -0.246 to -0.053$)$. The groups had similar respiratory symptoms. Catch-up growth for weight was defined as an increase in $z$ score of at least 0.67 between birth and ages $8-9$ years. For the IUGR children, 430 caught up and 146 did not. All spirometry measurements were higher in IUGR children who experienced catch-up growth than in those without, although the differences were not statistically significant. Both groups remained significantly lower than control subjects. Growth-retarded asymmetric and symmetric children had similar lung function.

Conclusion We concluded that IUGR is associated with poorer lung function at 8-9years of age compared with control children. Although the differences were not statistically significant, spirometry was higher in children who showed weight catch-up growth, but remained significantly lower than in the control children.

\section{THE EFFECTS OF PREGNANCY PLANNING, TIME TO CONCEPTION AND ART ON EXPRESSIVE LANGUAGE ABILITY AT YEARS}

doi:10.1136/jech.2010.120956.61

${ }^{1} \mathrm{C}$ Carson, ${ }^{2} \mathrm{Y}$ Kelly, ${ }^{1} \mathrm{~J} \mathrm{~J}$ Kurinczuk, ${ }^{3} \mathrm{~A}$ Sacker, ${ }^{1} \mathrm{M}$ Redshaw, ${ }^{1} \mathrm{M}$ A Quigley. ${ }^{1}$ National Perinatal Epidemiology Unit, University of Oxford, Headington, Oxford, UK; ${ }^{2}$ Department of Epidemiology and Public Health, University College London, London, UK; ${ }^{3}$ Institute of Social and Economic Research, University of Essex, Colchester, Essex, UK

Objective To examine the effects of pregnancy planning, time to conception and assisted reproductive treatment on expressive language ability at 3 years.

Design Population-based cohort (Sweeps 1 and 2 of the UK Millennium Cohort Study).

Setting UK.

Participants 11790 English-speaking singletons born 2000-2001.

Exposure measure Mothers reported whether the pregnancy was planned, and their feelings when first pregnant. Women who planned their pregnancy provided time to conception (TTC) and details of any assisted reproductive treatment (ART). The population was divided into "unintended" pregnancies (unplanned and unhappy about pregnancy), "unplanned" pregnancies (unplanned but happy), "planned" (planned and TTC $\leq 12$ months), "subfertile" (planned and TTC $>12$ months), "ovulation stimulated (OS)" (received clomiphene citrate) and "ART" (in vitro fertilisation or intracytoplasmic sperm injection).

Outcome measure Expressive language ability was assessed using the Naming Vocabulary component of the British Ability Scale. There is evidence that expressive language skills predict cognitive ability.

Results $15 \%$ of pregnancies were unintended and a further $26 \%$ were unplanned. $53 \%$ were planned; $4 \%$ subfertile; $2 \%$ used OS and $1 \%$ ART to help them conceive. The mean BAS naming vocabulary score was 76.5 in the planned group and was not significantly different in the OS or subfertile groups. In the unadjusted analysis 